\title{
奇宇称晶场和自旋-轨道耦合对钡铁氧体中 $\mathrm{Ni}^{2+}$ 离子磁光效应的影响
}

\author{
张锡娟 (1)*徐游(2) \\ (1)扬州大学理学院物理系, 扬州 225002; (2)南京大学物理系, 南京 210008 ; * 联系人)
}

摘要 用量子理论定量计算了进入 $2 \mathrm{~b}$ 座的 $\mathrm{Ni}^{2+}$ 离子 $\mathrm{d}-\mathrm{d}$ 跃迁造成的磁光偏转值, 结果表明掺 $\mathrm{Ni}$ 钡铁氧体的磁光偏转主要不是 d-d 跃迁造成的. 计算了 Faraday 旋转与基组态及激发组态 自旋-轨道耦合强度的关系, 其结果是基组态的自旋-轨道耦合对其磁光偏转起决定作用, 而激 发组态的影响不大, 且磁光偏转与基组态自旋-轨道耦合强度不成正比.

\section{关键词 Faraday 旋转 自旋-轨道耦合 奇宇称晶场 电偶极跃迁}

磁光效应是研究磁性材料的电子结构及磁性离子行为的非常有用的工具. 同时, 它又有 许多很重要的技术应用, 如磁光记录、电磁波传播器等. 作为第一代磁光存储材料的稀土一 过渡金属合金仍有其缺点. 近年实验发现, 用 $\mathrm{Ni}, \mathrm{Co}$ 置换后的钡铁氧体出现大磁光效应 ${ }^{[1,2]}$. $\mathrm{Ba}_{1-x} \mathrm{M}_{x} \mathrm{Fe}_{12-x} \mathrm{O}_{19}$ (这里 $\mathrm{M}$ 是 $\mathrm{La}$ 或 $\mathrm{Pr}$ ) 膜的 $496 \mathrm{~nm}$ 波长 (2.5eV) Faraday 旋转达 $2 \times 10^{4}$ $\left({ }^{\circ}\right) \mathrm{cm}^{-1} / x^{[1]}$. 由于钡铁氧体具有较大的单轴各向异性, 其磁滞回线的矩形度较好, 因此, 含 $\mathrm{Ni}$ 钡铁氧体引起了广泛的注意. 在我们之前, 常将铁氧体中 $3 \mathrm{~d}$ 离子的磁光效应归因为两种 机理: (1) 奇宇称晶场引起的 $d-d$ 电偶极跃迁; (2)3d 轨道与近邻离子的 $2 \mathrm{P}$ 轨道 (如氧离子) 或 $6 \mathrm{P}$ 轨道 (如铋离子) 的杂化效应所引起的 $3 \mathrm{~d}-2 \mathrm{p}$ (或 $6 \mathrm{p}$ ) 电偶极跃迁. 但还只有定性分析, 未见 有定量的计算 ${ }^{[2 \sim 4]}$. 我们近年的工作 ${ }^{[5]}$ 提出了一个新的模型, 即离子内 $3 \mathrm{~d}^{n} \rightarrow 3 \mathrm{~d}^{n-1} 4 \mathrm{p}$ 电偶 极跃迁. 该模型较好地解释了掺 $\mathrm{Ni}$ 钡铁氧体中大磁光偏转. 为了研究奇宇称晶场所引起的 d-d 跃迁导致的 Faraday 旋转究竟有多大, 是否也是含 $\mathrm{Ni}$ 钡铁氧体大磁光效应的一个起源, 本 文用量子理论定量计算了进入 $2 \mathrm{~b}$ 座的 $\mathrm{Ni}^{2+}$ 离子 $\mathrm{d}-\mathrm{d}$ 跃迁造成的磁光偏转值. 计算值比实验 值小一个数量级, 说明掺 $\mathrm{Ni}$ 钡铁氧体磁光偏转主要不是 $\mathrm{d}-\mathrm{d}$ 跃迁造成的.

早在 1932 年, Hulme ${ }^{[6]}$ 就提出, 对于铁磁材料, 激发态的自旋-轨道耦合对于产生磁光效 应是必需的. 1988 年后, Misemer 和 Oppeneer 等人经过定量计算, 指出 $\mathrm{MnBi}^{[7]}$ 和 $\mathrm{Fe}, \mathrm{Co}$, $\mathrm{Ni}^{[8]}$ 的磁光效应与自旋-轨道耦合强度几乎成线性关系. Dionne 等人 ${ }^{[9]}$ 则认为含 $\mathrm{Bi}$ 石榴石的 大磁光偏转主要是激发组态的能级在自旋-轨道耦合的作用下产生大的䢃裂而造成的. 为了 探究自旋-轨道耦合在 Faraday 旋转中的作用, 本文用量子理论计算了 Faraday 旋转与基组态 及激发组态自旋-轨道耦合强度的关系.

电偶极跃迁产生的 Faraday 旋转由下式给出 ${ }^{[10]}$

$$
\theta_{\mathrm{F}}=\frac{N \pi e^{2}(\bar{n}+2)^{2}}{9 \bar{n}^{2} c \hbar} \sum \frac{\omega^{2}}{\omega_{n g}^{2}-\omega^{2}}\left\{\left|\left\langle n\left|V_{-}\right| g\right\rangle\right|^{2}-\left|\left\langle n\left|V_{+}\right| g\right\rangle\right|^{2}\right\} \rho_{g},
$$

各量的意义见文献 [10]. 晶体中 $\mathrm{Ni}^{2+}$ 离子的 Hamilton 包含 $H_{0}, H_{\mathrm{SO}}, H_{\mathrm{C}}, H_{\mathrm{ex}} 4$ 项, 其中 $H_{\mathrm{SO}}$ 为自旋-轨道耦合作用, $H_{0}+H_{\mathrm{SO}}$ 为自由离子的 Hamilton, $H_{\mathrm{C}}$ 为晶场作用, $H_{\mathrm{ex}}$ 是超交换作 用. 通过解下列久期方程: 


$$
\left\|\left\langle L, J, J_{Z}\left|H_{0}+H_{\mathrm{SO}}+H_{\mathrm{C}}\right| L^{\prime}, J^{\prime}, J_{Z}{ }^{\prime}\right\rangle-E^{0} \delta_{L L^{\prime}} \delta_{J J^{\prime}} \delta_{J J_{Z}}{ }^{\prime}\right\|=0,
$$

可得 $\mathrm{Ni}$ 离子经晶场和自旋-轨道堣合作用后䢃裂的能级和波函数. 作用于 $\mathrm{Ni}$ 离子的超交换 作用引起的 Zeeman 效应可由下式解出 :

$$
\left\|\left\langle\varphi_{i}\left|H_{0}+H_{\mathrm{SO}}+H_{\mathrm{C}}+H_{\mathrm{ex}}\right| \varphi_{j}\right\rangle-E \delta_{i j}\right\|=0,
$$

其中 $\left|\varphi_{i}\right\rangle$ 为 $(2)$ 式得到的本征态.

\section{1 奇宇称晶场引起的 d-d 跃迁}

已知 ${ }^{[5]}$ 当 $\mathrm{Ni}$ 离子进入铁氧体中的 $2 \mathrm{~b}$ 座以后所引起的 Faraday 旋转较大. $2 \mathrm{~b}$ 座不具有中 心对称, 因而存在奇宇称晶场, 故本文内容都是考虑 $\mathrm{Ni}$ 离子进入 $2 \mathrm{~b}$ 座的情况. 根据点电荷模 型和 Slater 径向波函数计算出偶宇称和奇宇称晶场系数. 在解方程 (2) 时, 其中 $H_{\mathrm{C}}$ 包含了奇 宇称晶场和偶宇称晶场, 左、右矢分别包括 $\mathrm{Ni}$ 离子的 $3 \mathrm{~d}^{8}$ 组态中的 ${ }^{3} \mathrm{~F}$ 和 ${ }^{3} \mathrm{P}$ 项以及 $3 \mathrm{~d}^{7} 4 \mathrm{p}$ 组态 中的 ${ }^{3} \mathrm{D},{ }^{3} \mathrm{~F},{ }^{3} \mathrm{P} 3$ 个谱项。这 3 个谱项经晶场和自旋-轨道耦合䢃裂后能量最低的 19 个能级的 主要成分为 $3 \mathrm{~d}^{8}$ 态, 最低能级 $\left(-4721.4 \mathrm{~cm}^{-1}\right)$ 为二重简并态, 其主要成分为 $3 \mathrm{~d}^{8}{ }^{3} \mathrm{~F}, 4 \mathrm{p}$ 的成分 很小. 由于其他能级的占有几率均很小, 计算 Zeeman 效应时, 只需考虑最低一条能级. 解方 程(3)得到它的 2 条子能级分别为 $-5178 \mathrm{~cm}^{-1}$ 和 $-4264 \mathrm{~cm}^{-1}$. 由(1)式计算 Faraday 旋转 时, $\langle g|$ 只包括这 2 条能级, $|n\rangle$ 态则是高于这两条能级的所有 $3 \mathrm{~d}^{8}$ 组态的能级; 根据选择规 则, 对 Faraday 旋转有贡献的共有 8 条能级, 其中 2 条能级 $\left(13739 \mathrm{~cm}^{-1}\right.$ 和 $\left.17513 \mathrm{~cm}^{-1}\right)$ 的跃迁 对奇宇称晶场产生的 Faraday 旋转的贡献最大. 由于最低的晶场能级的主要成分是 $3 \mathrm{~d}^{8}{ }^{3} \mathrm{~F}$, 上 述 2 条较高能级的主要成分是 $3 \mathrm{~d}^{8}{ }^{3} \mathrm{P}$, 但其中包含了较大的 $4 \mathrm{p}$ 成分. 故其间的跃迁通常称为 $3 \mathrm{~d}^{3} \mathrm{~F} \rightarrow 3 \mathrm{~d}^{3} \mathrm{P}$ 跃迁, 简称为 $\mathrm{d}-\mathrm{d}$ 跃迁. 经具体计算, $2 \mathrm{~b}$ 座 $\mathrm{Ni}^{2+}$ 中, 由奇宇称晶场引起的 $\mathrm{d}-\mathrm{d}$ 跃迁 产生的室温 $496 \mathrm{~nm}$ Faraday 旋转为 $611.8\left(^{\circ}\right) \mathrm{cm}^{-1} / x$. 比实验值 $2 \times 10^{4}\left({ }^{\circ}\right) \mathrm{cm}^{-1} / x$ 小了近 2 个数量级. 因而含 $\mathrm{Ni}$ 钡铁氧体大磁光偏转的主要原因不是奇宇称晶场引起的. 在这个计算 结果中 $3 d^{7} 4 p$ 组态的谱项只考虑了最低的 3 个, $3 d^{7}\left({ }^{4} \mathrm{~F}\right) 4 \mathrm{p},{ }^{3} \mathrm{~F},{ }^{3} \mathrm{D},{ }^{3} \mathrm{P}$, 更高能量的自旋三重谱 项由于更远离 $3 d^{8}$ 组态, 在 $3 d^{8}$ 组态的能级中所含这些谱项的成分会更小. 我们相信由这些 谱项的 d-d 跃迁所造成的 Faraday 旋转值决不会超过 $611.8\left(^{\circ}\right) \mathrm{cm}^{-1} / x$ 的 1 倍, 因而不影响我 们所给出的结论.

\section{2 自旋-轨道耦合对磁光效应的影响}

在考虑自旋-轨道耦合的作用时, 只考虑偶宇称晶场的作用, 忽略了奇宇称晶场. 在 $3 \mathrm{~d}^{8}$ 组态向 $3 d^{7} 4 p$ 组态跃迁时, 包括了 $3 d^{7} 4 p$ 组态的全部 15 个自旋三重态谱项的贡献, $3 d^{7}\left({ }^{4} \mathrm{~F}\right)$ $4 \mathrm{p}:{ }^{3} \mathrm{~F},{ }^{3} \mathrm{D},{ }^{3} \mathrm{P}$, 这 3 个谱项的能量为 $110000 \mathrm{~cm}^{-1}$ 左右, 各谱项间的能量间隙很小, 在计算它们 在偶宇称晶场作用下的䢃裂时, 考虑了各谱项由于晶场引起的混合. 而较高的 12 个谱项的能 量在 $130000 \mathrm{~cm}^{-1}$ 左右, 且实验未能得到其准确数值. 我们在计算中将这些谱项的能量全部 认为等于 $130000 \mathrm{~cm}^{-1}$, 忽略其晶场䢃裂.

我们定义 $\xi_{1}\left(\xi_{2}\right)$ 为 $3 d^{8}\left(3 d^{7} 4 p\right)$ 组态自旋-轨道耦合强度系数. 当 $\xi_{1}, \xi_{2}$ 在 $0 \sim 4$ 范围中变 化时, 室温下 Faraday 旋转计算值, 列于表 1 .

由表可见, 当 $\xi_{2}$ 保持为 1 而 $\xi_{1}$ 变化时, Faraday 旋转值变化很大, 但与文献 [7], [8]所得 的 $\mathrm{MnBi}, \mathrm{Fe}, \mathrm{Co}, \mathrm{Ni}$ 金属 Faraday 旋转与自旋-轨道耦合作用成正比的结果不同: 当 $\xi_{1}$ 为零时, 
Faraday 旋转值几乎为零; 随着 $\xi_{1}$ 增加 Faraday 偏转迅速增大, 且当 $\xi_{1}$ 为 0.5 时几乎达饱和, 以后变化缓慢. 其原因是在 $\xi_{1}=1$ 时, 最低的第 1 条晶场能级和第 3 条能级对 Faraday 旋转的 贡献相反, 第 2 条能级对 Faraday 旋转几乎无贡献, 但此时 3 条能级之间的间距较大, 第 3 能 级的占有几率接近 0 , 故 Faraday 旋转很大. 随着 $\xi_{1}$ 值的减少, 3 条能级的间距变小, 第 1 、 第 3 条能级的占有几率接近, 故Faraday旋转值变小. 当 $\xi_{1}$ 等于零时, 则 3 条能级合为 1 条

表 1 室温下 Faraday 旋转计算值随 $\xi_{1}, \xi_{2}$ 变化(单位: $\left({ }^{\circ}\right) \mathrm{cm}^{-1} / x$ )

\begin{tabular}{|c|c|c|c|c|c|c|}
\hline \multicolumn{2}{|c|}{ 光能/eV } & 1 & 2 & 2.5 & 4 & 6 \\
\hline$\xi_{2}=1$ & $\xi_{1}=0$ & -11 & -48 & -77 & -231 & -737 \\
\hline$\xi_{2}=1$ & $\xi_{1}=0.25$ & 1520 & 6140 & 9665 & 25551 & 61478 \\
\hline$\xi_{2}=1$ & $\xi_{1}=0.50$ & 2344 & 9474 & 14918 & 39514 & 95537 \\
\hline$\xi_{2}=1$ & $\xi_{1}=1$ & 2399 & 9692 & 15256 & 40351 & 97220 \\
\hline$\xi_{2}=1$ & $\xi_{1}=2$ & 2844 & 10945 & 14559 & 53296 & 128277 \\
\hline$\xi_{2}=1$ & $\xi_{1}=4$ & 2759 & 10726 & 15467 & 55024 & 128382 \\
\hline$\xi_{1}=1$ & $\xi_{2}=0$ & 2406 & 9721 & 15304 & 40500 & 97720 \\
\hline$\xi_{1}=1$ & $\xi_{2}=0.25$ & 2404 & 9713 & 15292 & 40461 & 97597 \\
\hline$\xi_{1}=1$ & $\xi_{2}=0.50$ & 2403 & 9706 & 15280 & 40424 & 97472 \\
\hline$\xi_{1}=1$ & $\xi_{2}=1$ & 2399 & 9691 & 15256 & 40351 & 97220 \\
\hline$\xi_{1}=1$ & $\xi_{2}=2$ & 2392 & 9662 & 15208 & 40200 & 96700 \\
\hline$\xi_{2}=1$ & $\xi_{2}=4$ & 2378 & 9600 & 15106 & 39883 & 95595 \\
\hline
\end{tabular}

六重简并能级, Faraday 效应几乎完全抵消. 应当指出, 当上述 3 条能级的间距小到一定程度 时,我们的计算考虑了超交换作用所导致的它们之间的混合. $\xi_{2}$ 变化仅引起激发组态能级䢃 裂情况变化, 因而引起磁光共振频率改变, 但由于共振频率远离可见光频率, 故如表所示, 当保 持 $\xi_{1}$ 为 1 不变, 而 $\xi_{2}$ 在 $0 \sim 4$ 范围内变化时, Faraday 旋转的变化非常小. 由以上计算, 我们 得到如下结论:(1)基组态的自旋-轨道耦合对含 $\mathrm{Ni}$ 钡铁氧体中的磁光偏转起着决定的作用, 如果没有基组态的自旋-轨道耦合,则 Faraday 旋转也几乎为零, 但 Faraday 偏转与基组态自旋 一轨道耦合强度不成正比; (2) 激发组态的自旋-轨道耦合对 Faraday 旋转的影响不大, 因此 Dionne 等人 ${ }^{[9]}$ 提出的设想(仅有定性讨论,未经定量计算), 未必是正确的.

致谢 本工作为江苏省教委及国家自然科学基金资助(批准号:19674023)资助项目。

\section{参考文 献}

1 Gomi M, Shimai $\mathrm{K}$, Cho J, et al. Microstructure and $\mathrm{MO}$ enhancement in Ni-substituted Ba ferrite films prepared by sputtering. J Appl Phys, 1993, 73: 6145

2 Abe M, Gomi M. Magneto-optic Kerr effect in $\mathrm{CoFe}_{2-x} \mathrm{M}_{x} \mathrm{O}_{4}(\mathrm{M}=\mathrm{Mn}, \mathrm{Cr}, \mathrm{Al})$ and $\mathrm{BaFe}_{12-2 x}-\mathrm{Co}_{x} \mathrm{Ti}_{x} \mathrm{O}_{19}$ with tetrahedrally coordinated $\mathrm{Co}^{2+}$. J Appl Phys, 1982, 53: 8172

3 Shiragawa K, Tamanoi K, Saito T, et al. Cotton-Mouton effect of $\mathrm{CO}^{2+}$ substituted magnetic garnets. J Phys (Pavis), 1988, 49 C8: 9597

4 Ahrenkiel R K, Lyn S L, Coburn T J. Reflectance-circular dichroism of the magnetic insulator $\mathrm{Co}_{x} \mathrm{Cd}_{1-x} \mathrm{Cr}_{2} \mathrm{~S}_{4}$. J Appl Phys, $1975,46: 894$

5 Zhang Xijuan, Xu You, Duan Mingqian, et al. An investigation on the magnetooptical enhancement in Ni-substituted barium ferrites. J Appl Phys, 1996, 79(8) : 5979 
6 Hulme H R. The Faraday effect in ferromagnetics. Proc R Soc Lindon, 1932, A135: 237

7 Misemer D K. The effect of spin-orbit interaction and exchange splitting on magneto-optical coefficients. J Magn Magn Mater, $1988,72: 267$

8 Opperear P M, Sticht J, Maurer T, et al. Ab initio investigation of microscopic enhancement facters in tuning the magnetooptical kerr effect. Physik B, 1992, 88: 309

9 Dionne Gerald F, Allen Gary A. Molecular-orbital analysis of magneto-optical Bi-O-Fe hybrid excited states. J Appl Phys, $1994,75(10): 6372$

10 Crossley W A, Cooper R W, Page J L, et al. Faraday rotation in rare-earth iron garnets. Phys Rev, 1969, $181: 896$

(1998-01-05 收稿, 1998-08-10 收修改稿)

\section{毛细管内流动的近似分析模型}

\section{丁国良 张春路 李 影 陈芝久}

(上海交通大学制冷与低温工程系, 上海 200030)

摘要 通过积分方法建立了毛细管内流动的近似分析模型. 模型覆盖了毛细管内流动可能出 现的过冷、两相和过热 3 种流动区域，给出了不同流动区域的近似积分解. 用此模型预测了制 冷剂 R12, R134a 和 R600a 通过毛细管的质流率, 并与分布参数模型的结果比较, 平均偏差小 于 $1 \%$, 最大偏差 $2.2 \%$, 计算速度提高 1 个数量级以上.

\section{关键词 毛细管 模型 制冷剂}

毛细管作为节流元件在制冷空调装置中得到广泛使用, 其流动特性对装置性能影响显 著, 因此建立精确, 同时又简单、通用的毛细管模型对于实际装置的设计与优化具有重要意 义. 由于管内流体流动的高度非线性, 各种较为精确的分布参数模型在数值求解时速度较慢 且存在计算的稳定性问题 ${ }^{[1,2]}$. 而本文通过分区积分法建立的毛细管内流动的近似分析模 型，计算速度快，而且避免了计算的不稳定。

\section{1 毛细管的分区积分模型}

对于一般的制冷循环分析, 制冷剂在毛细管内的流动可认为是定常的一维等焓均相流 动, 有如下控制方程：

$$
-\mathrm{d} p=G^{2} \mathrm{~d} v+\frac{1}{2} \frac{f}{D} v G^{2} \mathrm{~d} L,
$$

式中 $p, v, G$ 分别为流体的压力、比容和质流密度, $D$ 和 $L$ 分别为毛细管内径和长度, $f$ 为 沿程摩阻系数.

\section{1 过冷区模型}

过冷区液体比容和沿程摩阻系数可认为不变, 对(1)式积分, 得过冷区长度

$$
L_{\mathrm{SC}}=\frac{2 \Delta p_{\mathrm{SC}} D}{f_{\mathrm{SC}} v_{\mathrm{SC}} G^{2}}
$$

式中, $\Delta p_{\mathrm{SC}}$ 表示过冷区压降, 下标 SC 表示过冷区. 